\title{
Proteinuria modifies the effects of physical activity on total and cardiovascular disease mortality rates in patients with type 2 diabetes
}

\author{
T. Vepsäläinen • M. Soinio • S. Lehto • A. Juutilainen • \\ M. Laakso • T. Rönnemaa
}

Received: 5 March 2010/Accepted: 11 May 2010/Published online: 6 June 2010

(C) Springer-Verlag 2010

\begin{abstract}
Aims/hypothesis Physical activity reduces cardiovascular disease (CVD) and total mortality rates in patients with type 2 diabetes. However, it is not known whether or not the effects of physical activity on mortality rates depend on the presence of proteinuria in type 2 diabetic patients. Methods We prospectively followed up 577 patients with type 2 diabetes who were aged 45 to 64 years and were free of CVD at baseline. Participants were stratified according to the presence of proteinuria $(\leq 300$ or $>300 \mathrm{mg} / \mathrm{l})$ and the degree of physical activity $(0-4$ metabolic equivalent tasks [MET] or $>4$ MET). The Cox proportional hazards model was used to estimate the association of physical activity and proteinuria with risk of mortality.

Results During the 18-year follow-up, 356 patients died, of whom 217 died from CVD. Physically more active patients had significantly reduced total, CVD and CHD mortality rates if they did not have proteinuria. In contrast, physically active proteinuric patients had significantly increased total and CVD mortality rates (HR 1.83, 95\% CI 1.00-3.36, $p=0.049)$ in univariate analyses, with HR $2.43(95 \%$ CI $1.09-5.40, p=0.030$ ) in multivariate analyses.
\end{abstract}

T. Vepsäläinen • T. Rönnemaa $(\bowtie)$

Department of Medicine, University of Turku,

Kiinamyllynkatu 4-8,

20520 Turku, Finland

e-mail: tapani.ronnemaa@utu.fi

M. Soinio

Department of Medicine, Turku University Hospital,

Turku, Finland

S. Lehto $\cdot$ A. Juutilainen $\cdot$ M. Laakso

Department of Medicine,

University of Kuopio and Kuopio University Hospital,

Kuopio, Finland
Conclusions/interpretation Physical activity reduces total and CVD mortality rates in type 2 diabetic patients without proteinuria. However, in proteinuric patients, no protective effect was observed. Larger studies are needed to confirm the latter finding and to define which exercise intensity leads to possible harmful effects.

Keywords Cardiovascular disease - Physical activity . Type 2 diabetes
Abbreviations
CVD Cardiovascular disease
MET Metabolic equivalent task

\section{Introduction}

Patients with type 2 diabetes have an approximately threefold risk of cardiovascular disease (CVD) death compared with non-diabetic individuals [1]. Regular physical activity is associated with reduced CVD and total mortality rates in the general population [2]. In addition to CVD, diabetes is often associated with microvascular complications, e.g. retinopathy and nephropathy. Proteinuria is a strong and independent predictor of CVD and total mortality rates in type 2 diabetes [3]. Diabetic microvascular complications and macrovascular disease share similar pathogenetic features, and proteinuria and microalbuminuria have been proposed to be a sign of widespread vascular damage [4].

Although physical activity is considered a cornerstone in hyperglycaemia treatment and prevention of vascular events in diabetic patients [5], more research is needed to confirm the overall health benefits of exercise and to provide more accurate exercise guidelines, especially in diabetic patients with complications [6]. Therefore, we investigated the 
association between physical activity and both CVD and total mortality rates among type 2 diabetes patients with and without proteinuria in a large population-based 18-year follow-up study.

\section{Methods}

Patients A more detailed description of study participants has been given elsewhere [1]. Briefly, 1,059 Finnish type 2 diabetic patients aged 45 to 64 years were identified. Type 1 diabetic patients were excluded on the basis of the following criteria: early onset of diabetes, ketoacidosis history and glucagon-stimulated C-peptide measurement at baseline.

We excluded 452 participants from statistical analyses because they had angina pectoris, possible or definite stroke, possible or definite myocardial infarction, intermittent claudication or amputation at the baseline; we also excluded 30 participants who died or had a severe CVD event (myocardial infarction, stroke or lower limb amputation) during the first two follow-up years. Exclusion criteria were based on the assumption that participants had changed their exercise habits due to severe disease at baseline. Thus, the final study population consisted of 577 type 2 diabetic patients (314 men, 263 women). Due to missing proteinuria data, we additionally excluded three patients from proteinuria analyses.

Baseline study The baseline examinations between 1982 and1984 included an interview on smoking history, alcohol intake, physical activity, medication and history of chest pain suggestive of CHD using the Rose cardiovascular questionnaire. Medical records of hospitalised chest pain patients were reviewed. WHO criteria for verified definite or possible myocardial infarction based on chest pain symptoms, ECG changes and enzyme determinations were used to define previous myocardial infarction. ECG abnormalities were classified by Minnesota code. WHO criteria were also used to define previous definite or possible stroke. Hypertension was defined as systolic BP $\geq 160 \mathrm{mmHg}$ or diastolic BP $\geq 95 \mathrm{mmHg}$ or antihypertensive drug treatment. The methods have been described previously in detail [1].

Biochemical methods After a $12 \mathrm{~h}$ fast, blood specimens were taken at 08:00 hours. $\mathrm{HbA}_{1}$ (non-diabetic reference range 5.5$8.5 \%$ ) was determined by affinity chromatography (Isolab, Akron, OH, USA). Serum total cholesterol, HDL-cholesterol and triacylglycerol were determined with standard laboratory methods as described previously [1]. Coomassie brilliant blue method was used to measure total urinary protein concentration from a morning spot urine specimen [7].

Assessment of physical activity Metabolic equivalent task (MET) was used to assess physical activity. One
MET corresponds to $3.5 \mathrm{ml}$ of oxygen uptake $(\mathrm{kg}$ bodyweight $)^{-1} \mathrm{~min}^{-1}$. For example, 4 METs requires four times the metabolic energy expenditure of that when the person is sitting quietly, and is equivalent to brisk walking.

At the baseline examination, occupational, commuting and leisure-time physical activity was assessed using a selfadministered questionnaire. A MET value was assigned for each of the three categories according to the intensity of the activity [8]. We used the highest intensity of occupational, commuting or leisure-time activity to represent overall activity level of the participants.

The reliability of the questionnaire on physical activity has been evaluated in 1,400 Finnish individuals. The reliability estimated by kappa coefficients was for most questions at least 0.6 [8].

Follow-up study The follow-up period lasted until 1 January 2001. Copies of death certificates of deceased participants were obtained from the Cause-of-Death Register (Statistics Finland). In the final classification of causes of death, hospital and autopsy records were also used if available. The endpoints used in this study were total, CHD (ICD-9 codes 410-414) and CVD (ICD-9 codes 390-459) death.

The Ethics Committees of Kuopio University Hospital and Turku University Central Hospital approved the study. All study participants gave informed consent.

Statistical analyses Statistical analyses were performed using SPSS 15.0 (SPSS, Chicago, IL, USA). Data for continuous variables are expressed as means $\pm \mathrm{SD}$ and categorical variables as percentages. Student's $t$ test for independent samples was used to assess group differences. Triacylglycerol values were analysed after logarithmic transformation. The $\chi^{2}$ test was used to compare categorical variables. The Cox proportional hazards model was used to estimate single and joint association of physical activity level and proteinuria category with mortality risk. Participants were classified according to physical activity (0-4 MET, $>4$ MET) and proteinuria $(\leq 300$ or $>300 \mathrm{mg} / \mathrm{l})$. Unadjusted and adjusted HRs and their 95\% CIs were calculated. Because no interactions between sex and physical activity or proteinuria were observed, men and women were combined for analysis. A $p$ value of $p<0.05$ was considered statistically significant.

\section{Results}

Physically active type 2 diabetic patients were slightly younger, had lower triacylglycerol levels and lower BMI, smoked less often, were less likely to have hypertension and received insulin therapy more often than physically inactive patients (Table 1). 
Table 1 Baseline characteristics with various outcomes according to physical activity level among type 2 diabetic patients
Data are expressed as the mean \pm $\mathrm{SD}$, unless otherwise indicated

\begin{tabular}{|c|c|c|c|}
\hline \multirow[t]{2}{*}{ Characteristic } & \multicolumn{2}{|c|}{ Physical activity } & \multirow[t]{2}{*}{$p$ value } \\
\hline & 0-4 MET & $>4 \mathrm{MET}$ & \\
\hline$n$ & 347 & 230 & \\
\hline Age (years) & $58.2 \pm 5.1$ & $56.5 \pm 5.3$ & $<0.001$ \\
\hline Duration of diabetes (years) & $7.9 \pm 4.0$ & $7.8 \pm 4.0$ & 0.689 \\
\hline Total cholesterol (mmol/l) & $6.62 \pm 1.68$ & $6.39 \pm 1.38$ & 0.089 \\
\hline HDL-cholesterol (mmol/l) & $1.24 \pm 0.35$ & $1.28 \pm 0.36$ & 0.195 \\
\hline Triacylglycerol (mmol/l) & $2.39 \pm 2.58$ & $1.89 \pm 1.24$ & 0.006 \\
\hline $\mathrm{HbA}_{1}(\%)$ & $9.7 \pm 1.8$ & $9.9 \pm 3.0$ & 0.222 \\
\hline BMI $\left(\mathrm{kg} / \mathrm{m}^{2}\right)$ & $29.9 \pm 5.9$ & $28.2 \pm 4.4$ & $<0.001$ \\
\hline Women (\%) & 50.1 & 38.7 & 0.007 \\
\hline Current smokers (\%) & 19.6 & 11.3 & 0.008 \\
\hline Hypertension (\%) & 61.7 & 52.2 & 0.024 \\
\hline Alcohol users (\%) & 37.5 & 42.6 & 0.216 \\
\hline Diabetes treatment & & & 0.054 \\
\hline Diet only $(\%)$ & 13.0 & 16.1 & \\
\hline Oral glucose-lowering drugs (\%) & 76.9 & 68.3 & \\
\hline Insulin therapy $(\%)$ & 10.1 & 15.7 & \\
\hline Proteinuria $(n)$ & & & 0.287 \\
\hline No & 288 & 200 & \\
\hline Yes & 56 & 30 & \\
\hline \multicolumn{4}{|l|}{ Participants with endpoints $(n)$} \\
\hline Death from all causes & 234 & 122 & \\
\hline Death from any CVD & 143 & 74 & \\
\hline Death from CHD & 98 & 51 & \\
\hline
\end{tabular}

In the physically active group, 25 of 30 proteinuric participants died (16 from CVD), while 97 of 200 participants without proteinuria died (58 from CVD). In the physically inactive group, 45 of 56 proteinuric participants died (26 from CVD) and 187 of 288 participants without proteinuria (116 from CVD).
The physically active group without proteinuria had a significantly reduced risk of death from CVD, CHD and all causes in univariate and multivariate analyses. There were no significant differences between physically active and inactive proteinuric patients in univariate analyses, but in multivariate analyses physically active patients with pro-

Table 2 Hazard ratios (physically active vs inactive) for CVD, CHD and total mortality rates stratified by proteinuria

\begin{tabular}{|c|c|c|c|c|}
\hline \multirow[t]{2}{*}{ Variable } & \multicolumn{2}{|l|}{ Relative risk, no adjustment } & \multicolumn{2}{|c|}{ Relative risk, multivariable adjustment ${ }^{\mathrm{a}}$} \\
\hline & Active vs inactive HR $(95 \% \mathrm{CI})$ & $p$ value & Active vs inactive HR $(95 \% \mathrm{CI})$ & $p$ value \\
\hline \multicolumn{5}{|l|}{ CVD mortality } \\
\hline No proteinuria & $0.59(0.43-0.80)$ & 0.001 & $0.63(0.45-0.88)$ & 0.007 \\
\hline Proteinuria & $1.24(0.66-2.31)$ & 0.500 & $2.43(1.09-5.40)$ & 0.030 \\
\hline \multicolumn{5}{|l|}{ CHD mortality } \\
\hline No proteinuria & $0.62(0.42-0.90)$ & 0.011 & $0.66(0.44-0.97)$ & 0.037 \\
\hline Proteinuria & $1.09(0.49-2.45)$ & 0.833 & $1.49(0.51-4.38)$ & 0.472 \\
\hline \multicolumn{5}{|l|}{ Total mortality } \\
\hline No proteinuria & $0.61(0.48-0.78)$ & $<0.001$ & $0.66(0.51-0.86)$ & 0.002 \\
\hline Proteinuria & $1.11(0.68-1.81)$ & 0.688 & $1.83(1.00-3.36)$ & 0.049 \\
\hline
\end{tabular}

${ }^{a}$ Variables in multivariable adjustment: age, sex, diabetes duration, $\mathrm{HbA}_{1}$, total cholesterol, presence of hypertension, smoking, alcohol, BMI, area of residence, type of diabetes therapy, triacylglycerol and HDL-cholesterol 
teinuria had a significantly increased risk of death from CVD and all causes (Table 2).

\section{Discussion}

Our study is the first population-based study to show that physical activity significantly reduces the risk of death from CVD, CHD and all causes in type 2 diabetes patients without proteinuria. In patients without proteinuria, the beneficial effect of physical activity on mortality rates was independent of conventional CVD risk factors, glycaemic control, diabetes duration and treatment. In proteinuric patients, a protective effect of physical activity was lost. In fact, increased physical activity was associated with higher total and CVD mortality rates, suggesting that physical activity may even be harmful in these patients.

Several mechanisms could explain why physical activity reduces CVD and total mortality rates. Physical activity has favourable effects on insulin sensitivity, $\mathrm{HbA}_{1 \mathrm{c}}$, weight loss, hypertension, serum lipid profile and maximal exercise capacity in patients with type 2 diabetes. Physical activity might also reduce inflammation, cytokine response and oxidative stress $[6,9]$.

In our study, proteinuric patients did not benefit from physical activity. Even though our study has limited statistical power because of the smaller number of proteinuric patients, several mechanisms suggest that our findings are likely to be valid. First, diabetic patients with long-term complications have impaired total antioxidant status compared with patients without complications [10]. It is possible that in proteinuric patients exercise enhances superoxide production, which together with already diminished antioxidant enzyme levels could result in accelerated atherogenesis and increased CVD event risk. Furthermore, both exaggerated blood pressure response to exercise, which is known to occur in patients with albuminuria, and sympathetic overactivation related to acute exercise could also contribute to increased mortality rates.

Our study has several strengths. First, it was not until the later half of the 1990s that statin treatment became widespread. Therefore, it is unlikely that lipid-lowering therapy could have caused major bias. Second, unlike many other similar studies, we had data on glucose control, diabetes duration and mode of treatment. Third, we excluded type 1 diabetic patients from statistical analyses. Finally, to avoid potential bias from possibly increased early mortality rates due to a severe disease at baseline in patients with low physical activity, we excluded all participants with a prior diagnosis of CVD and those who died or experienced a severe disability during the first 2 years of follow-up.
Our study also has some limitations. We measured total urinary protein concentration from spot urine samples instead of measuring urinary AER. Our cut-off point for proteinuria, $300 \mathrm{mg} / \mathrm{l}$, corresponds to the common lower value used for proteinuria. Therefore, our findings do not apply to patients with microalbuminuria. Another limitation is that we only evaluated physical activity at baseline. It is likely that these limitations of our study underestimate rather than overestimate the association of physical activity and proteinuria with cardiovascular outcomes and total mortality rates.

In conclusion, physical activity reduced cardiovascular and total mortality rates in middle-aged type 2 diabetic participants without proteinuria, whereas this beneficial effect was not observed in patients with proteinuria. The observation that physical activity in proteinuric patients may actually be harmful needs to be confirmed by larger studies and information on characteristics of possibly harmful exercise defined before being translated into exercise guidelines.

Duality of interest The authors declare that there is no duality of interest associated with this manuscript.

\section{References}

1. Laakso M, Rönnemaa T, Pyörälä K, Kallio V, Puukka P, Penttilä I (1988) Atherosclerotic vascular disease and its risk factors in noninsulin dependent diabetic and non-diabetic subjects in Finland. Diabetes Care 11:449-463

2. Blair SN, Kohl HW III, Barlow CE, Paffenbarger RS Jr, Gibbons LW, Macera CA (1995) Changes in physical fitness and all-cause mortality: a prospective study of healthy and unhealthy men. JAMA 273:1093-1098

3. Miettinen H, Haffner SM, Lehto S, Rönnemaa T, Pyörälä K, Laakso M (1996) Proteinuria predicts stroke and other atherosclerotic vascular disease events in nondiabetic and non-insulin-dependent diabetic subjects. Stroke 27:2033-2039

4. Deckert T, Feldt-Rasmussen B, Borch-Johnsen K, Jensen T, Kofoed-Enevoldsen A (1989) Albuminuria reflects widespread vascular damage: the Steno hypothesis. Diabetologia 32:219-226

5. $\mathrm{Hu} \mathrm{G}$, Eriksson J, Barengo $\mathrm{NC}$ et al (2004) Occupational, commuting, and leisure-time physical activity in relation to total and cardiovascular mortality among Finnish subjects with type 2 diabetes. Circulation 110:666-673

6. Marwick TH, Hordern MD, Miller T et al (2009) Exercise training for type 2 diabetes mellitus: impact on cardiovascular risk: a scientific statement from the American Heart Association. Circulation 119:3244-3262

7. Van Kley H, Hale SM (1977) Assay for protein by dye binding. Anal Biochem 81:485-487

8. Mälkiä E, Impivaara $\mathrm{O}$, Maatela J, Aromaa A, Heliövaara M, Knekt P (1988) Physical activity of Finnish adults. Series ML 80. Social Insurance Institution, Turku

9. Kojda G, Hambrecht R (2005) Molecular mechanisms of vascular adaptations to exercise. Physical activity as an effective antioxidant therapy? Cardiovasc Res 67:187-197

10. Čolak E, Majkić-Singh N, Stanković S et al (2005) Parameters of antioxidative defense in type 2 diabetic patients with cardiovascular complications. Ann Med 37:613-620 